\title{
USE OF COMPUTER ASSISTANCE IN ORDER TO DESIGNATE THE TASKS IN THE MUNICIPAL SERVICES COMPANIES
}

\author{
Mariusz Izdebski, Marianna Jacyna \\ Warsaw University of Technology Faculty of Transport \\ Koszykowa Street 75, 00-662 Warsaw, Poland \\ tel.: +4822 2346017, fax: +48222346017 \\ e-mail:izdebski.mariusz@interia.pl,maja@wt.pw.edu.pl
}

\begin{abstract}
In this article, the method of designating the tasks in the municipal services companies was described. Presented method consists of three phase: the preparatory phase, the optimization phase and the generated tasks phase. Each phase was characterized. In this paper, the mathematical model of this problem was presented. The function of criterion and the condition on designating the tasks were defined. The minimum route described in the optimization phase was designated by the genetic algorithm. In this paper, the stages of constructing of the genetic algorithm were presented. A structure of the data processed by the algorithm, a function of adaptation, a selection of chromosomes, a crossover, a mutation and an inversion were characterized. A structure of the data was presented as string of natural numbers. In selection process, the roulette method was used and in the crossover, process the operator PMX was presented. The method was verified in programming language $C$ \#. The process of verification was divided into two stages. In the first stage, the best parameters of the genetics algorithm were designated. In the second stage, the algorithm was started with these parameters and the result was compared with the random search algorithm. The random search algorithm generates 2000 routes and the best result is compared with the genetic algorithm. The influence of the inversion, the mutation and the crossover on quality of the results was examined.
\end{abstract}

Keywords: municipal services companies, transport, optimization, genetic algorithm, verification

\section{Introduction}

The main task of the municipal services companies is the waste collection from the region. The issue of the waste collection is a complex decision problem, which relates to the traveling salesman problem [11]. One of the main problems in the municipal services companies is the vehicle routing [2]. This issue is difficult to solve because of many points of the waste collection, the limit of working time of a driver, the limit of driving time of a driver, the limit of time of tasks realization.

The method of designating the route of vehicles proposed by authors of this publication consists of two stages. The first stage relies on designating the tasks in companies; the second stage relies on allocating vehicles to these tasks. The stage of designating the tasks relies on indicating the point of beginning of the task and the point of ending of the task. The point of ending we can define as the point where the vehicle leaves the route and goes to the dumping ground. These points start and end the route of the waste collection realized by vehicles. Complexity of this stage relies on that each point of loading is located in the different place. The task is designated when we know the route of the vehicles, which collect the waste, the point of beginning of the route, the points of the route and the point of ending of the route. The stage of allocation is also a complex stage, which is realized after ending the first stage.

\section{The method of designating the tasks in the municipal services companies}

The presented method consists of three phases: the preparatory phase, the optimization phase and the generated tasks phase. 
In the first phase, all data are introduced to the method in order to its implementation e.g. the number of points of loading, the volume of waste in the point of loading, payload of the vehicle. The main task of this phase is to determine so - called: collective points of loading. Collective points of loading (on the Fig. 1) we create by allocating individual points of loading to such sections of the road where the vehicles on these sections cannot change their route. The volume of this point cannot exceed payload of the vehicle.

The main task of the second phase is to designate the minimum route consisting of all collective points of loading. Designating this route is a complex optimization problem, a decision variable appears when the vehicle commutes to the junction and then the length of the entire route depends on the decision of the driver.

The main task of the third phase is to divide the minimum route into the segments, which are interpreted as the tasks. The beginning of the segment is defined as the point where the vehicle starts the waste collection, the ending of the segment is defined as the point where the vehicle leaves the route and goes to the dumping ground. The division of the minimum route depends on payload of the vehicle, which realizes this route. The minimum route was designated be means of the genetic algorithm.

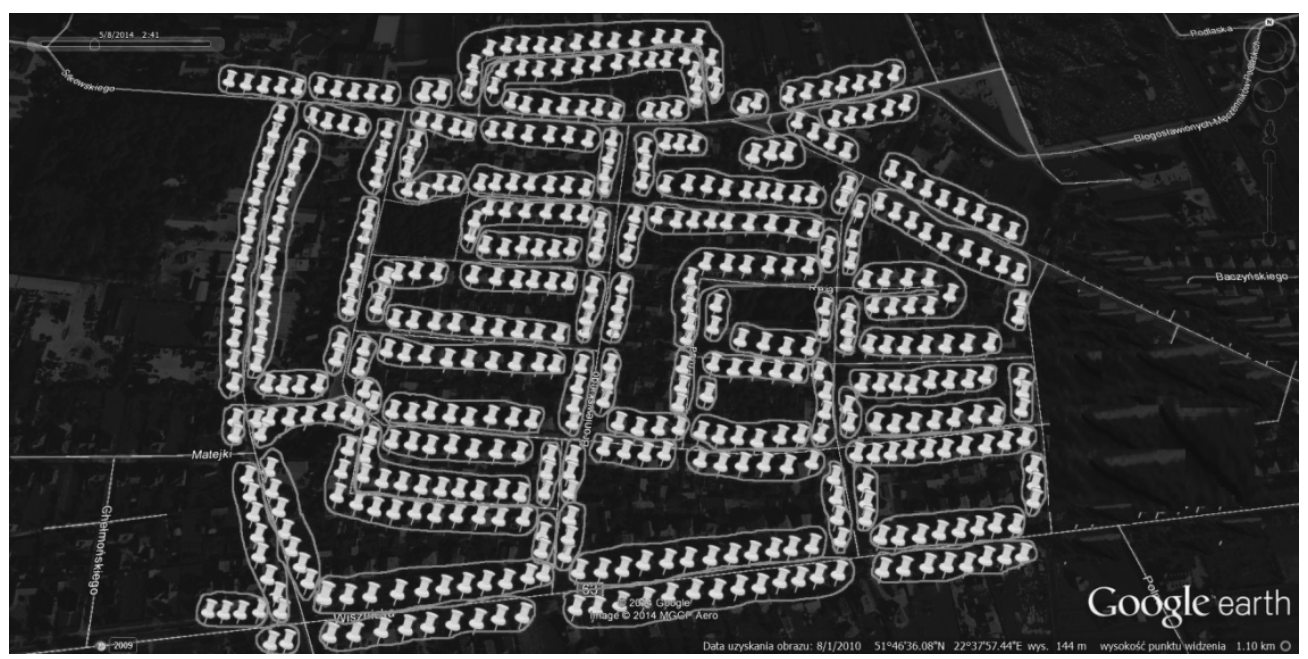

Fig. 1. Collective points of loading in the area of the waste collection

The vehicle visits collective points of loading and collects the waste and when payload of the vehicle is exceeded, the vehicle leaves the route. The nature of functioning of the municipal services companies makes, that we cannot designate clearly and permanently number of tasks for implementation. This number is variable and it depends on payload of the vehicle, which was used to designate tasks. The vehicles in the companies have different payload and the minimum route can be divided into the different number of segments according to payload of the vehicles, which were used to designate this tasks. At first, the minimum route is divided by the vehicles of maximum payload and thereby we have a larger number of collective points of loading realized by the vehicles and a smaller number of designated tasks. Such designated tasks can be realized only by vehicles of maximum payload. In the case where the number of vehicles is too small to realize these tasks, the stage of designating the tasks must be repeated again. For the remaining collective points of loading, we designate the minimum route again and we divide this route using the vehicle of smaller payload. The stage of designating the tasks we repeat until the moment of realization all collective points of loading.

\section{The mathematical model of designating the tasks}

The task of the vehicle is to visit all collective points of loading where the order of visits influences the length of the route. In order to determine the mathematical model the following variables were used [8]: 


$$
\begin{aligned}
& \begin{array}{l}
W^{Z} \quad \text { the set of collective points of loading, } W^{Z}=\left\{1, \ldots, i, j, \ldots, \overline{W^{Z}}\right\}, \\
\mathbf{D}=[d(i, j)] \quad \text { the matrix of the distance between } i-\text { this collective point loading and } \\
\quad j-\text { this collective point of loading, where } i \neq j,
\end{array} \\
& \mathbf{X T}=[x t(i, j)]- \\
& \begin{aligned}
\text { binary matrix of a decision variable defining the road between } i-\text { this collective } \\
\text { point of loading and } j \text { - this collective point of loading, where } i \neq j .
\end{aligned}
\end{aligned}
$$

The function of criterion minimizes the total length of the route and takes the form:

$$
F(\mathbf{X T})=\sum_{i \in W^{z}} \sum_{j \in W^{z}} x t(i, j) \cdot d(i, j) \rightarrow \min .
$$

After determining the minimum route, we can go to determine the tasks through designating the points where the vehicle leaves the route. These points will divide the route into small segments, which are interpreted as the tasks. The vehicle goes to the dumping ground when it is not able to load another collective point of loading on the route; the loading is limited by payload of the vehicle. Let us assume that $p(n)$-payload $n$ - of this vehicle, where $n \in N, N$ - the set of vehicles, $p z(k)$ - the volume of waste in the $k$ - this collective point of loading, where $k \in K, K-$ the set of collective points of loading.

We can write down the condition on designating the tasks:

$$
\begin{gathered}
\forall n \in N, \\
\sum_{i \in W^{Z}} \sum_{j \in W^{Z}} \sum_{k \in K} x t(i, j) \cdot p z(k) \leq p(n) .
\end{gathered}
$$

\section{The algorithm of designating the minimum route}

The minimum route was designated by the genetic algorithm. Genetic algorithms are algorithms which activity is based on the mechanisms of natural selection and heredity. They are used as a functional and practical optimization tool $[3,4,7,9]$. Methods of this type give a nearoptimum solution, can find the optimal solution but often confine themselves to the optimal solution in the local area of search. Despite this inconvenience, genetic algorithms are successfully used in optimization problems. The genetic algorithm consists of the following steps:

- Step 1 - The determining of a structure of the data processed by the algorithm,

- Step 2 - The determining of a function of adaptation,

- Step 3 - The selection of chromosomes dependent on the function of adaptation,

- Step 4 - The crossover of chromosomes selected randomly out of the pool of parent,

- Step 5 - The mutation of chromosomes,

- Step 6-The inversion.

The steps from three to six of the genetic algorithm are repeated until the stop condition is achieved. The stop condition in this algorithm is a predetermined number of generations (iterations).

The basic structure of the input data for the classical genetic algorithm is a zero-one sequence. In this problem, the chromosome is represented by the string of natural numbers. Such structure of the input data successfully works out in similar issues where the use of zero-one strings significantly hinders the operation of the genetic algorithm, e.g. in the traveling salesman problem $[5,6,10]$. The genetic algorithm does not work directly on the decision variables of the function of criterion but on the encoded forms of these variables. In order to encode the variables of the function of criterion in an appropriate structure and create the chromosome as a representative of the admissible solution the problem of designating the minimum route must be defined as the appointment of the suitable permutation of collective points of loading so that their location would generate the minimum value of the function of criterion, which in our case it is the minimum length of the route. The task of the genetic algorithm is to find the best set of collective points of loading by 
optimizing function of adaptation. The structure of data suitable for processing by the genetic algorithm can be defined as a string consisting of $k$ collective point of loading. The total length of the chromosome is $k$ genes. Each chromosome is a representative of the transport route (Fig. 2).

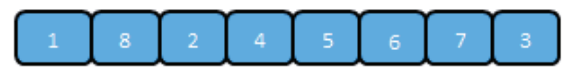

Fig. 2. The Structure of the chromosome in the genetic algorithm

The genetic algorithm works on the encoded forms of the decision variables. In order to designate the value of the $l$-this chromosome the function of adaptation $F_{l}^{\prime}$ was determined and it takes the form:

where:

$$
F_{l}^{\prime}=C_{\max }-F_{l}(X T)
$$

$C_{\max } \quad$ - the value larger than the value of chromosome. The maximum value of the function to adaptation $F^{\prime}$ is equal to the minimum value of the function of criterion,

$F_{l}(X T)$ - the real value of the function of adaptation for $l$ - this chromosome, which is equal to the function of criterion for each route.

The operation of reproduction (selection) consists of duplication of chromosomes depending on the function of adaptation. The chromosomes with the higher function of adaptation are more likely to introduce their own copy to the next generation. In selection process, the roulette method was used based on the selection of a new population according to the probability distribution defined on the values of the function of adaptation.

- The selection process consists of the following stages:

- the calculation of the function of adaptation for a single chromosome,

- the calculation of the total population of adaptation,

- the calculation of the probability of the selection $l$ of the chromosome,

- the calculation of the distribution $l$ of the chromosome.

Choosing the chromosome to the next generation consists of the random selection of the number $r$ from the range of $[0,1]$. We choose $l$ - the chromosome with the value of distribution $q_{l}$ while the relationship $q_{l-1}<r \leq q_{l}$ is fulfilled.

In the crossover operation an operator, which works on the structure of the numeric strings, was used, which is called PMX (partially matched crossover). The PMX [1], crossover is the random selection of the two chromosomes in pairs, random selection of the two points of crossover and exchange of genes shown by the series created from these points of crossover. In the process of crossover, genes from one chromosome are assigned to genes from another. The crossover occurs with probability $p^{k}$. The PMX crossover is shown in Fig. 3.

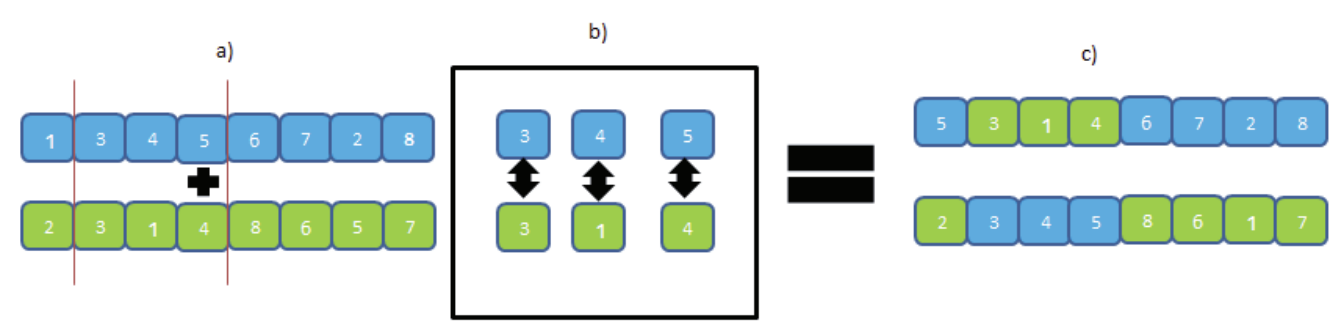

Fig. 3. The PMX crossover a) the chromosomes used to the crossover b) a swap of genes c) the chromosomes after crossover

A mutation is the swap of place of two randomly selected tasks. The mutation occurs with probability $p^{m}$.The principle of the mutation is shown in Fig. 4. 


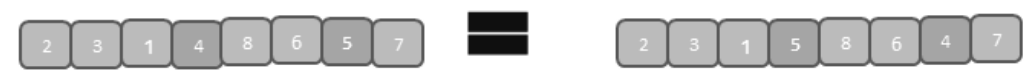

Fig. 4. The use of a mutation operator

The initial stage of inversion is a random selection of two points in the chromosome. These points create the string, which needs to be reversed. The inversion occurs with probability $p^{\text {in }}$. The principle of the inversion is shown in Fig. 5.

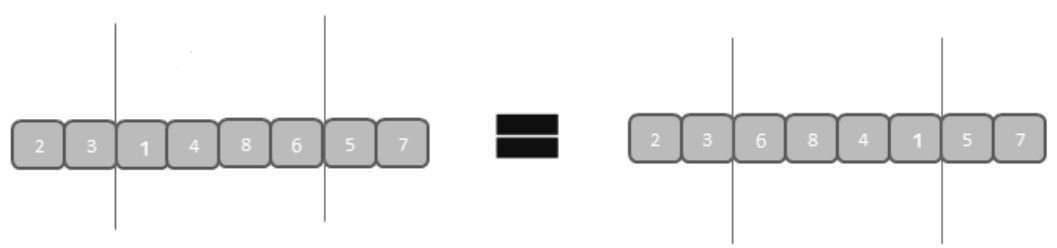

Fig. 5. The principle of the inversion

\section{Verification of the method}

The method was verified using programming language $\mathrm{C}$ \#. Verification of the method takes place in the phase of designating the minimum route and relies on comparing the result of the genetic algorithm with the result of the random search algorithm. The random search algorithm generates 2000 routes and the best result is compared with the genetic algorithm. The method was verified for 92 collective points of loading. The area of the waste collection was presented in the first section of this paper. The genetic algorithm was started 50 times so the result is the average of all starts. The number of iterations is equal to 200 , the population is 100 . The parameters of the algorithm take the values: $p^{k}=0,2 ; 0,4 ; 0,6 ; 08 ; 1, p^{m}=0,01 ; 0,03 ; 0,05, p^{i n}=0 ; 0,4 ; 0,6 ; 0,8 ; 1$.

75 combinations of these parameters were checked and the best combination of parameters, where the algorithm gave the best solution, was found. Combination of parameters was shown in Tab. 1.

Tab. 1. Combination of parameters in the genetic algorithm

\begin{tabular}{|c|c|c|c|c|c|c|c|c|c|c|c|}
\hline Lp. & $p^{k}$ & $p^{m}$ & $p^{\text {in }}$ & Lp. & $p^{k}$ & $p^{m}$ & $p^{\text {in }}$ & Lp. & $p^{k}$ & $p^{m}$ & $p^{\text {in }}$ \\
\hline 1 & 0.2 & 0.01 & 0 & 26 & 0.4 & 0.05 & 0 & 51 & 0.8 & 0.03 & 0 \\
\hline 2 & 0.2 & 0.01 & 0.4 & 27 & 0.4 & 0.05 & 0.4 & 52 & 0.8 & 0.03 & 0.4 \\
\hline 3 & 0.2 & 0.01 & 0.6 & 28 & 0.4 & 0.05 & 0.6 & 53 & 0.8 & 0.03 & 0.6 \\
\hline 4 & 0.2 & 0.01 & 0.8 & 29 & 0.4 & 0.05 & 0.8 & 54 & 0.8 & 0.03 & 0.8 \\
\hline 5 & 0.2 & 0.01 & 1 & 30 & 0.4 & 0.05 & 1 & 55 & 0.8 & 0.03 & 1 \\
\hline 6 & 0.2 & 0.03 & 0 & 31 & 0.6 & 0.01 & 0 & 56 & 0.8 & 0.05 & 0 \\
\hline 7 & 0.2 & 0.03 & 0.4 & 32 & 0.6 & 0.01 & 0.4 & 57 & 0.8 & 0.05 & 0.4 \\
\hline 8 & 0.2 & 0.03 & 0.6 & 33 & 0.6 & 0.01 & 0.6 & 58 & 0.8 & 0.05 & 0.6 \\
\hline 9 & 0.2 & 0.03 & 0.8 & 34 & 0.6 & 0.01 & 0.8 & 59 & 0.8 & 0.05 & 0.8 \\
\hline 10 & 0.2 & 0.03 & 1 & 35 & 0.6 & 0.01 & 1 & 60 & 0.8 & 0.05 & 1 \\
\hline 11 & 0.2 & 0.05 & 0 & 36 & 0.6 & 0.03 & 0 & 61 & 1 & 0.01 & 0 \\
\hline 12 & 0.2 & 0.05 & 0.4 & 37 & 0.6 & 0.03 & 0.4 & 62 & 1 & 0.01 & 0.4 \\
\hline 13 & 0.2 & 0.05 & 0.6 & 38 & 0.6 & 0.03 & 0.6 & 63 & 1 & 0.01 & 0.6 \\
\hline 14 & 0.2 & 0.05 & 0.8 & 39 & 0.6 & 0.03 & 0.8 & 64 & 1 & 0.01 & 0.8 \\
\hline 15 & 0.2 & 0.05 & 1 & 40 & 0.6 & 0.03 & 1 & 65 & 1 & 0.01 & 1 \\
\hline 16 & 0.4 & 0.01 & 0 & 41 & 0.6 & 0.05 & 0 & 66 & 1 & 0.03 & 0 \\
\hline 17 & 0.4 & 0.01 & 0.4 & 42 & 0.6 & 0.05 & 0.4 & 67 & 1 & 0.03 & 0.4 \\
\hline 18 & 0.4 & 0.01 & 0.6 & 43 & 0.6 & 0.05 & 0.6 & 68 & 1 & 0.03 & 0.6 \\
\hline 19 & 0.4 & 0.01 & 0.8 & 44 & 0.6 & 0.05 & 0.8 & 69 & 1 & 0.03 & 0.8 \\
\hline 20 & 0.4 & 0.01 & 1 & 45 & 0.6 & 0.05 & 1 & 70 & 1 & 0.03 & 1 \\
\hline 21 & 0.4 & 0.03 & 0 & 46 & 0.8 & 0.01 & 0 & 71 & 1 & 0.05 & 0 \\
\hline 22 & 0.4 & 0.03 & 0.4 & 47 & 0.8 & 0.01 & 0.4 & 72 & 1 & 0.05 & 0.4 \\
\hline 23 & 0.4 & 0.03 & 0.6 & 48 & 0.8 & 0.01 & 0.6 & 73 & 1 & 0.05 & 0.6 \\
\hline 24 & 0.4 & 0.03 & 0.8 & 49 & 0.8 & 0.01 & 0.8 & 74 & 1 & 0.05 & 0.8 \\
\hline 25 & 0.4 & 0.03 & 1 & 50 & 0.8 & 0.01 & 1 & 75 & 1 & 0.05 & 1 \\
\hline
\end{tabular}


Verification of the method consists of two stages. In the first stage, the best parameters of algorithm are designated. In the second stage, the algorithm is started with these parameters and the result is compared with the random search algorithm. In the first stage the influence of the inversion, the crossover and the mutation on quality of the results are examined. The influence of the inversion, the mutation and the crossover on quality of the results was shown in Fig. 6-8.

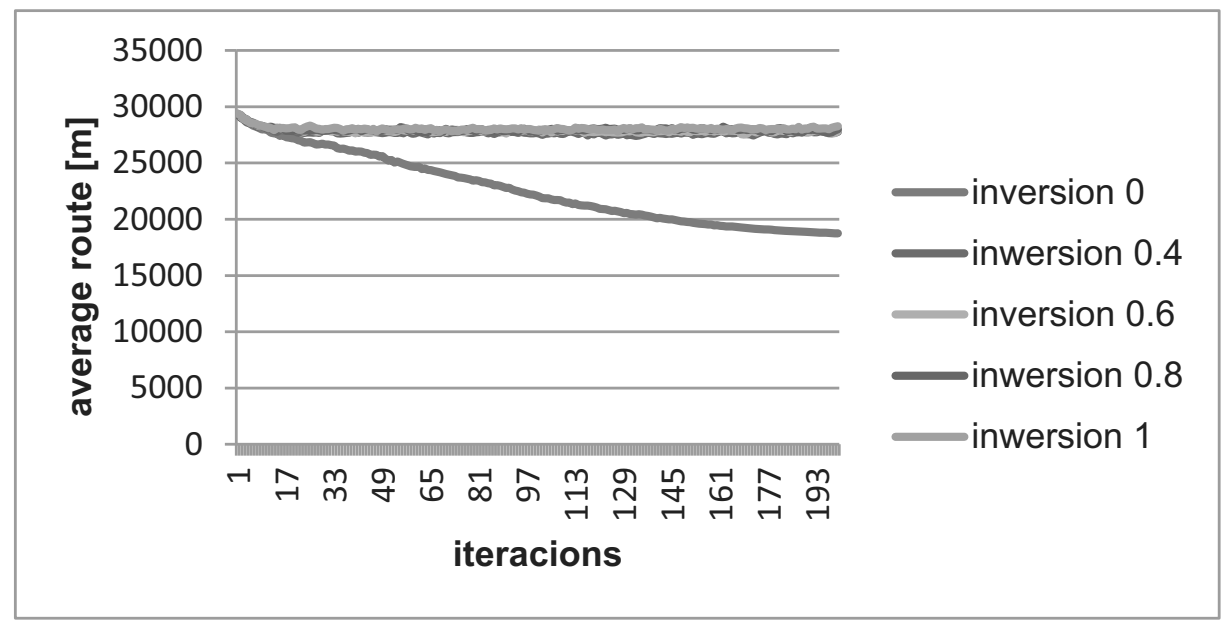

Fig. 6. The influence of the inversion on quality of the results

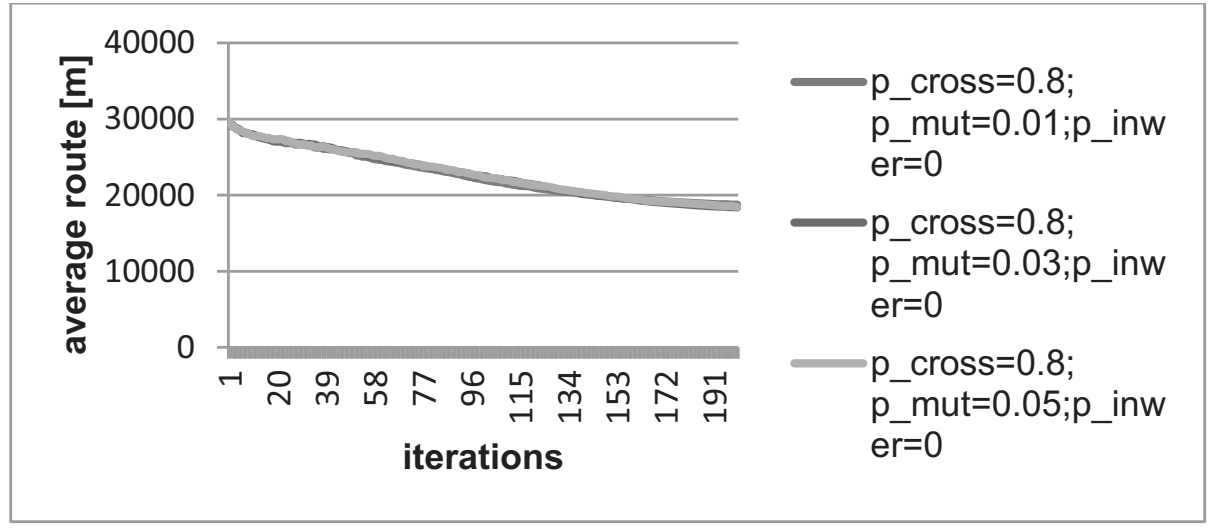

Fig. 7. The influence of the mutation on quality of the results

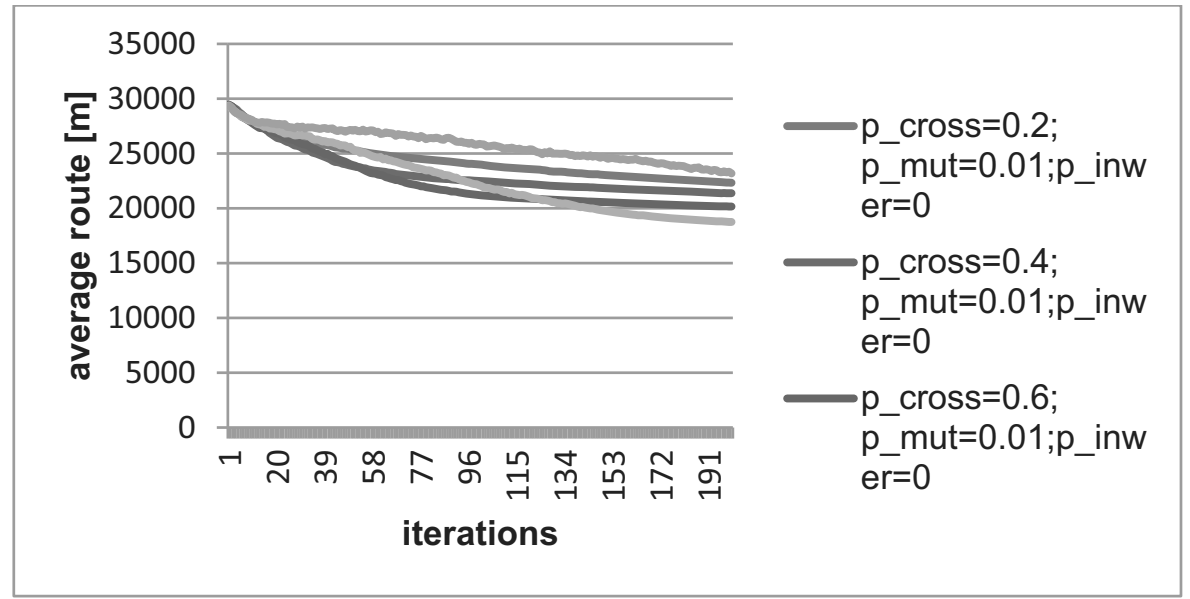

Fig. 8. The influence of the crossover on quality of the results

The best result was designated for the parameters: $p^{k}=1, p^{m}=0.03, p^{i n}=0$. In the second stage the algorithm was started with these parameters and the result was compared with the random search algorithm. The result was shown in Fig. 9. 


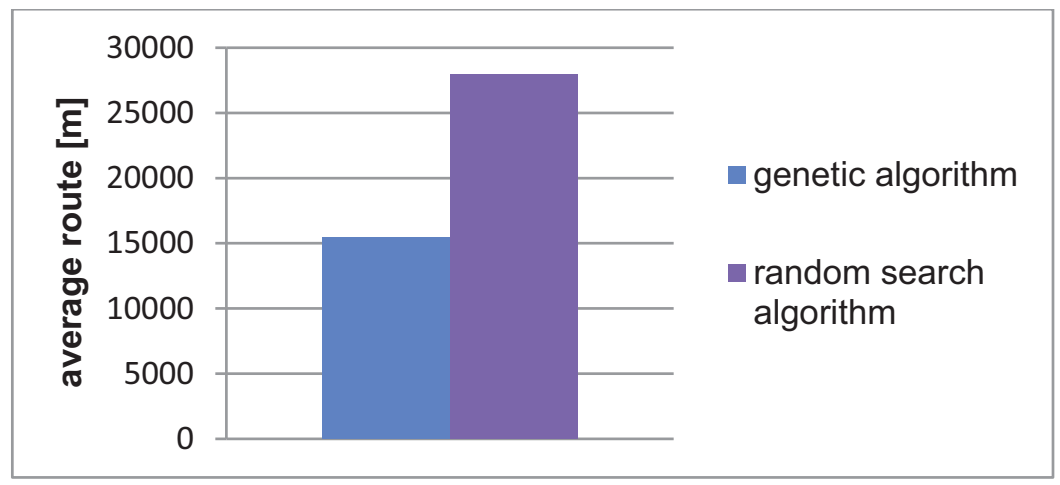

Fig. 9. Comparison of algorithms

\section{Summary}

Presented method of designating the tasks was verified in programming language $\mathrm{C} \#$. The most important stage of this method is the stage of designating the minimum route, which consists of collective points of loading. The length of the generated route influences the number of tasks; therefore, verification of the method must be done in this stage. After analysing the results of the genetic algorithm, we have come to conclusion that the inversion in each case does not improve quality of the solution. The increase of the crossover improves the quality of the solution. For 200 iteration for $p^{k}=1$ we cannot observe the convergence of the algorithm to a specified value, so the number of iterations was increased to 400 . Then the algorithm gave the best solution for all parameters of the crossover. The mutation has a minimum influence on quality of the results. The result is a combination of three parameters and it is very important to perform the stage of designating these parameters.

\section{References}

[1] Abdoun, O., Abouchabaka, J., A Comparative Study of Adaptive Crossover Operators for Genetic Algorithms to Resolve the Traveling Salesman Problem, International Journal of Computer Applications, Foundation of Computer Science, Vol. 31, No. 11, pp. 49-57, New York, USA 2011.

[2] Beliën, J., Boeck L., Municipal Solid Waste Collection and Management Problems: A Literature Revie, Transportation Science, Institute for Operations Research and the Management Sciences (INFORMS), Vol. 48, Is. 1, pp. 78-102, USA 2014.

[3] Bräysy, O., Gendreau M., Vehicle Routing Problem with Time Windows, Part II: Metaheuristics, Transportation Science, Institute for Operations Research and the Management Sciences (INFORMS), Vol. 39, Is. 1, pp. 119-139, USA 2005.

[4] Goldberg, D. E., Algorytmy genetyczne i ich zastosowanie, Wydawnictwo Naukowo-Techniczne, Warszawa 1995.

[5] Goldberg, D. E., Lingle, R., Alleles, Loci, and the TSP, Proceedings of the First International Conference on Genetic Algorithms, Lawrence Erlbaum Associates, Hillsdale, pp. 154-159, NJ 1985.

[6] Grefenstette, J. J.,Gopal, R., Rosmaita, B., Van Gucht, D., Genetic Algorithm for the TPS, Proceedings of the First International Conference on Genetic Algorithms, Lawrence Erlbaum Associates, Hillsdale, pp. 160-168, NJ 1985.

[7] Grzymkowski, R., Kaczmarek, K., Kiełtyka, S., Nowak, I., Wykłady z modelowania matematycznego, Wydawnictwo Pracowni Komputerowej Jacka Skalmierskiego, Gliwice 2008.

[8] Jacyna, M., Modelowanie $i$ ocena systemów transportowych, Oficyna Wydawnicza Politechniki Warszawskiej, Warszawa 2009. 
[9] Michalewicz, Z., Algorytmy genetyczne + struktury danych = programy ewolucyjne, Wydawnictwo Naukowo-Techniczne, Warszawa 1996.

[10] Nagata, Y., Kobayashi, S., A Powerful Genetic Algorithm Using Edge Assembly Crossover for the Traveling Salesman Problem, Transportation Science, Institute for Operations Research and the Management Sciences (INFORMS), Vol. 25, Is. 2, pp. 346-363, USA 2013.

[11] Płaczek, E., Szołtysek, J., Wybrane metody optymalizacji systemu transportu odpadów komunalnych w Katowicach, LogForum, Wyższa Szkoła Logistyki, Vol. 4, pp. 1-10, Poznan 2008. 